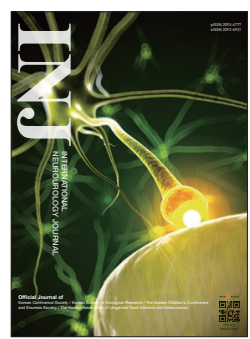

\title{
Possible Therapeutic Effect of Exercise for Incurable Diseases
}

\author{
Il-Gyu Ko (iD https://orcid.org/0000-0003-2696-6869 \\ Department of Physiology, College of Medicine, Kyung Hee University, Seoul, Korea \\ Email: rhdlfrb@hanmail.net
}

The topic of this special issue of the International Neurourology Journal is the "Possible therapeutic effect of exercise for incurable diseases." This special issue includes 5 papers on animal experiments and 2 papers on clinical trials. These papers focus on the therapeutic effects of several types of exercise on various difficult-to-treat diseases.

First, the animal experiments reported the following results. Cho et al. [1] found that treadmill exercise suppressed estrogen deficiency-induced apoptosis and the atrophy signaling pathway in rat skeletal muscle. Treadmill exercise also increased hypertrophy signaling in the skeletal muscles of rats. Kim et al. [2] showed the effects of resistance exercise on Parkinson disease in mice. Resistance exercise improved spatial learning ability by enhancing brain-derived neurotrophic factor expression through phosphorylation of 5'-adenosine monophosphate-activated protein kinase in Parkinson disease mice. Park et al. [3] suggested that maternal swimming exercise during pregnancy improved memory function by enhancing neurogenesis and suppressing apoptosis in autistic mice. This effect of swimming exercise appeared through activation of the Wnt $/ \beta$-catenin signaling pathway in valproic acid-injected pups. Lee et al. [4] discussed the effect of preischemic treadmill running on memory impairment in a rat model of chronic hypoperfusion. They concluded that preischemic treadmill exercise improved bilateral common carotid artery occlusion-induced spatial learning memory impairment. Preischemic treadmill exercise also ameliorated damage to the microvasculature and pericytes in the blood-brain barrier. Park et al. [5] demonstrated that poloxamer-407 injection induced hyperlipidemia and impaired short- term memory. Treadmill running improved short-term memory deficits in rats with poloxamer-407-induced hyperlipidemia by inhibiting neuroinflammation.

The clinical trial papers reported the following results. Ha and Sung [6] concluded that the Vojta approach is an intervention method that elicits a systemic motor response by stimulating a specific reflex zone in a specific starting position. The Vojta approach acted as an effective intervention method for improving neck stability and static balance in children with hypotonia. Lee and Jee [7] suggested that resistance training enhanced desirable changes in the adaptive immune cell response of ovarian cancer survivors by developing strength and endurance and maintaining skeletal muscle mass.

Exercise effectively prevents muscle atrophy by enlarging muscles, improves memory by increasing neurogenesis, and ameliorates degenerative disease symptoms by inhibiting apoptosis. We have collected only a few of the many therapeutic effects of exercise for intractable diseases. The research presented in this special issue on the positive effects of exercise on intractable diseases will provide an opportunity to discover new treatments for intractable urological diseases and the mechanisms through which urological disorders occur. On behalf of the editorial board, I am grateful to the authors for contributing their valuable articles to this issue.

- Conflict of Interest: No potential conflict of interest relevant to this article was reported. 


\section{REFERENCES}

1. Cho EJ, Choi Y, Kim J, Bae JH, Cho J, Park DH, et al. Exercise training attenuates ovariectomy-induced alterations in skeletal muscle remodeling, apoptotic signaling, and atrophy signaling in rat skeletal muscle. Int J Neurourol J 2021;25(Suppl 2):S47-54.

2. Kim SH, Hwang L, Jin JJ, Ko IG, Kim YB, Yoon HS, et al. Resistance exercise improves spatial learning ability through phosphorylation of 5'-adenosine monophosphate-activated protein kinase in Parkinson disease mice. Int J Neurourol J 2021;25(Suppl 2):S55-62.

3. Park SS, Kim CJ, Kim SH, Kim TW, Lee SJ. Maternal swimming exercise during pregnancy improves memory through enhancing neurogenesis and suppressing apoptosis via Wnt/ $\beta$-catenin pathway in autistic mice. Int J Neurourol J 2021;25(Suppl 2):S63-71.
4. Lee JM, Park J, Lee JH, Song MK, Kim YJ. Preischemic treadmill exercise ameliorates memory impairment and microvasculature damage in rat model of chronic cerebral hypoperfusion. Int J Neurourol J 2021;25(Suppl 2):S72-80.

5. Park SS, Kim TW, Sung YH, Park YJ, Kim MK, Shin MS. Treadmill exercise ameliorates short-term memory impairment by suppressing hippocampal neuroinflammation in poloxamer-407-induced hyperlipidemia rats. Int J Neurourol J 2021;25(Suppl 2):S81-9.

6. Ha SY, Sung YH. Vojta approach affects neck stability and static balance in sitting position of children with hypotonia. Int J Neurourol J 2021;25(Suppl 2):S90-5.

7. Lee JK, Jee YS. Effects of resistance exercise on acquired immunocytes in cancer survivors: a pilot study. Int J Neurourol J 2021;25 (Suppl 2):S96-105. 